\title{
Laryngeal Leishmaniasis
}

\section{Bruno Teixeira de Moraes', Francisco de Souza Amorim Filho², José Caporrino Neto 3 , Paulo Saraceni Neto ${ }^{4}$, José Elson Santiago de Melo Júnior.}

1) ENT. Master in Health Sciences Department of Otolaryngology and Head and Neck Surgery, Federal University of São Paulo.

2) Head and Neck Surgeon. PhD in Health Sciences Department of Otolaryngology and Head and Neck Surgery, Federal University of São Paulo.

3) ENT. Assistant Professor, Department of Otolaryngology and Head and Neck Surgery, Federal University of São Paulo.

4) Medical. Resident Department of Otolaryngology and Head and Neck Surgery, Federal University of São Paulo.

5) Physician. Specializing Department of Otolaryngology and Head and Neck Surgery, Federal University of São Paulo.

Institution: Division of Laryngology and Voice of the Department of Otolaryngology at the Federal University of São Paulo. São Paulo / SP - Brazil.

Correspondence to: Bruno Teixeira de Moraes - 957 Pedro de Toledo Street - Vila Clementino - São Paulo / SP - Brazil - Zip code: 04039032 - Telephone: (+55 11) 5573-2740 - E-mail: moraesbruno.orl@hotmail.com

Article submitted on August 7, 2010. Approved on October 24, 2010.

\section{SUMMARY}

Introduction: Leishmaniasis is classified into three clinical presentations: visceral, coetaneous and mucocutaneous. The latter is usually secondary to hematogenous spread after months or years of skin infection and can manifest as infiltrative lesions, ulcerated or vegetating in nose, pharynx, larynx and mouth, associated or not with ganglionics infarction. Laryngeal involvement is part of the differential diagnosis of lesions in this topography as nonspecific chronic laryngitis, granulomatosis and even tumors of the upper aerodigestive tract presenting atypical evolution. Sometimes it is difficult for the correct diagnosis of Leishmaniasis, with description of cases in the literature were conducted improperly.

Objective: The objective of this study is to report a case of laryngeal Leishmaniasis addressing the difficulty of diagnosis, complications and treatment applied.

Case Report: A patient with pain throat, dysphagia, odynophagia, dysphonia and weight loss, with no improvement with symptomatic medication. At telelaringoscopy, infiltrative lesion showed nodular supraglottis. He underwent a tracheotomy for airway obstruction and biopsy with immunohistochemical study for a definitive diagnosis of laryngeal Leishmaniasis. The patient was referred to the infectious diseases that initiated treatment with $\mathrm{N}$-methylglucamine antimoniate with satisfactory response to therapy.

Final Comments: Faced with a clinical suspicion of granulomatous diseases, it is essential to follow protocol laboratory evaluation associated with histological injury, to get a precise definition etiological without prolonging the time of diagnosis. Medical treatment for mucosal Leishmaniasis, recommended by the World Health Organization, was adequate in the case of laryngeal disorders, with complete resolution of symptoms.

Keywords: Leishmaniasis; mucocutaneous Leishmaniasis; chronic granulomatous disease; larynx.

\section{INTRODUCTION}

Leishmaniasis is an infectious granulomatous disease; non-contagious disease caused by protozoan species Leishmania brasiliensis, L. amazonensis, L. panamensis and Leishmania guyanensis, typically found in the Americas. Its transmission occurs through the bite of sandflies insects, whose genre most often found in Brazil is Lutzomya. Over the past 20 years, the occurrence of this disease is growing rapidly, both in magnitude and geographical expansion, with outbreaks in various regions of the country and, more recently, in the Amazon area, related to the predatory process of colonization (1).
Traditionally, the disease is classified into three clinical presentations: visceral (Kala-azar), coetaneous and mucocutaneous. The latter is usually secondary to hematogenous spread after months or years of skin infection and can manifest as infiltrative lesions, ulcerated or vegetating in nose (most common site), pharynx, larynx and mouth, bloating associated or not with lymph node (2). The involvement of mucosa is dependent on the combination of virulence of the parasite and cellular host immune response. Among the population of infected individuals, 1 to 10\% occurs with mucosal involvement; however, the specific factors that will determine which patients will develop the mucocutaneous Leishmaniasis are still not well understood (3). 
Low prevalence, laryngeal involvement Leishmaniasis is part of the differential diagnosis of lesions in this topography as nonspecific chronic laryngitis, and even granulomatosis of upper aerodigestive tract tumors that exhibit atypical evolution $(4,5)$. Sometimes it is difficult for the correct diagnosis of Leishmaniasis, with description of cases in the literature were conducted initially as malignancy, including undergoing radiotherapy, with subsequent confirmation of infectious etiology (6).

The objective of this study is to report a case of laryngeal Leishmaniasis addressing their diagnostic difficulty, complications and treatment applied.

\section{Case Report}

SJS patient, male, 51 years old, black, mason, born in União dos Palmares - AL, and living in Sao Paulo - SP, was treated at the Otolaryngology department of a tertiary hospital in São Paulo, with reports of "sore throat" there three years, which has evolved over the past four months with dysphagia, odynophagia, dysphonia and weight loss of about 8 pounds in this period, no improvement with symptomatic medication. He denied fever, ear or respiratory complaints. Smoking history presented as moderate, severe alcoholics without a history of hypertension, diabetes mellitus or infectious diseases.

On physical examination, with regular general condition, without significant changes in the respiratory, cardiovascular or abdomen. At telelaringoscopy, nodular infiltrative lesion was visualized in supraglottis involving the epiglottis, aryepiglottic, looking vegetating in left piriformis sinus and arytenoids edema. The vocal folds were mobile and without structural lesions with complete glottal closure (Figure 1).

Preoperative tests were ordered, then the patient underwent suspension laryngoscopy with biopsy of the lesion and the histopathology revealed the case of an ulcerated inflammatory process.

Three weeks after this biopsy, the patient developed dyspnea and worsening of dysphagia, accepting only pasty-liquid diet. Tracheotomy was performed and new biopsy showed that the atypical lymphoid infiltration histopathology. The piece was sent to perform immunohistochemistry to detect amastigote of Leishmania sp. in the sample.

The patient was referred to the infectious diseases that initiated treatment with $\mathrm{N}$-methylglucamine antimoniate at a dose of $20 \mathrm{mg} / \mathrm{kg} /$ day, intramuscularly, for

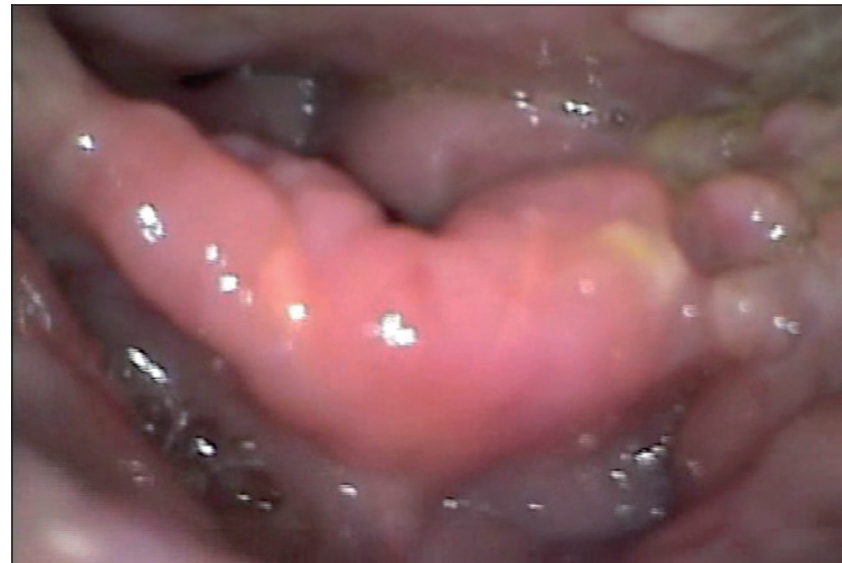

Figure 1. Laryngoscopy Pretreatment.

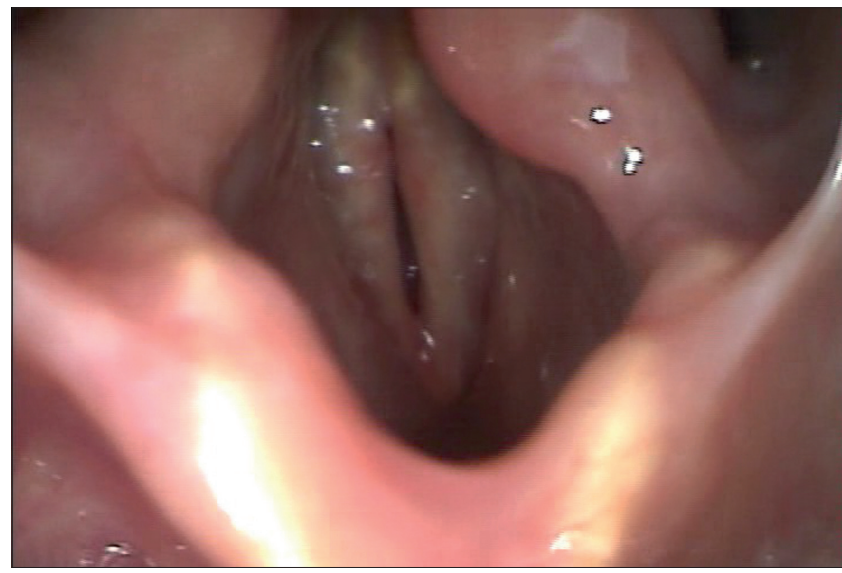

Figure 2. Laryngoscopy Post-treatment.

30 days with outpatient and uneventful. There was significant improvement of the table early in the treatment, control laryngoscopy after the 30th day showed resolution of the lesions. Then, the tracheotomy was performed decannulation (Figure 2).

Three months after treatment, the patient presented with significant improvement of his general condition and nutritional asymptomatic and in their professional activities.

\section{Discuss}

Leishmaniasis is rarely detected early on. The patient usually presents one or few skin lesions, which appear three to ten weeks after inoculation, with a tendency to spontaneous healing in a few months, even without treatment. In some cases the lesions remain active for several years and it is estimated that 3 to 5\% may develop with subsequent emergence of mucosal 
lesions by hematogenous spread. In the case reported the presentation was mucosa of unknown origin, since there was no coetaneous lesion. It is believed that such would probably be associated with subclinical infections or minor injuries, non-ulcerated, with rapid evolution and that would have gone unnoticed without noticeable scarring (1).

Pharyngolaryngeal involvement can be intense enough to cause dysphagia, dyspnea, dysphonia, sore throat and cough. A peculiarity of this case differs from that literature is the absence of nasal lesions which are often found in the initial shape of the mucosal Leishmaniasis. Even this is a parameter used in the differential diagnosis of diseases such as tuberculosis and laryngeal Paracoccidioidomycosis who rarely have associated nasal lesions (7).

The laryngoscopy usually demonstrates an extensive inflammatory component, with erythema and edema evident. The granulomatous associated ulcers are common and may also submit purulent exudates. In advanced disease, tissue destruction may be striking. Before this presentation, it is important hypotheses of other diseases as leprosy, sarcoidosis, syphilis, Wegner granulomatosis, systemiclupus erythematosus actinomycosis, Histoplasmosis and neoplasm $(8,9)$.

By presenting a more gorgeous, both in terms of symptomatology and the findings of laryngoscopy, the patient was referred initially reported to the laryngeal lesion biopsy in an attempt to elucidate the diagnosis, rather than a complement careful laboratory evaluation. Given that the diagnosis of Leishmaniasis can be determined by parasitological examinations (direct search or culture), immunological tests (intradermal of Montenegro) or molecular tests (polymerase chain reaction), solving this case likely would have been anticipated if they were requested Routine tests for suspected cases of granulomatosis (VDRL, Montenegro reaction, PPD, serology for Histoplasmosis and Paracoccidioidomycosis, chest radiography). Especially because the cases of mucosal Leishmaniasis usually have strong positive intradermal of Montenegro, and secondly parasitological confirmation difficult due to the shortage parasite, which was evident by the need for two biopsies and complementation analysis with immunohistochemistry for the identification of amastigote (1) . Therefore, as research protocol for suspected etiologic granulomatosis in organs such as the larynx difficult access, should initially be requested laboratory tests and imaging, and case stay uncertain diagnosis, biopsy is indicated for the histological lesions. If the appearance of the lesion suggests malignancy, research through noninvasive tests and invasive must occur simultaneously to avoid delay diagnosis.
In the case of infectious diseases with risk of contamination, the diagnosis should be established as soon as possible. The interval between onset of symptoms and diagnosis in a way reflects the ease of access to health services as well as efficiency in diagnosis.

The regimen used was satisfactory. The same is in accordance with the recommendations of the World Health Organization (WHO), which recommends $\mathrm{N}$ methylglucamine antimoniate as first choice at a dose of $20 \mathrm{mg} / \mathrm{kg} /$ day lasting at least 30 days. In patients with contraindications (pregnancy due to teratogenicity) or refractory to standard treatment, should be used amphotericin B (10).

\section{FINAL COMMENTS}

Before clinical suspicion with granulomatous diseases, it is vital following protocol associated with laboratory evaluation of histological injury to achieve a precise definition etiological without prolonging the time of diagnosis. Medical treatment for mucosal Leishmaniasis, recommended by WHO, was adequate in the case of laryngeal disorders, with complete resolution of symptoms.

\section{REFERENCES}

1. Manual de Vigilância da Leishmaniose Tegumentar Americana. $2^{\text {a }}$. ed. Brasília: Editora do Ministério da Saúde; 2007.

2. Grant A, Spraggs PD, Grant HR, Bryceson AD. Laryngeal leishmaniasis. J Laryngol Otol. 1994;108(12):1086-1088.

3. David CV, Craft N. Cutaneous and mucocutaneous leishmaniasis. Dermatol Ther. 2009;22(6):491-502.

4. Sizeland A. Leishmaniosis in third world. N Engl J Med. 1995;332(9): 610-1.

5. Lightfoot, Stanley A. J Am Board Fam Pract. 1997;10(5):374-6.

6. Ravisse P, Bensimon P, Lapicorey G. A case of laryngeal leishmaniasis with a long course. Bull Soc Pathol Exot Filiales. 1984 May-Jun;77(3):305-11.

7. Lessa MM et al. Leishmaniose Mucosa: aspectos clínicos e epidemiológicos. BrazJ Otorhinolaryngol. 2007;73(6):843847.

8. Carvalho T, Dolci JEL. Avaliação clínica da influência do 
uso de glucantine em pacientes com Leishmaniose nasal. Acta ORL. 2006;24(2):77-82.

9. Caporrino Neto J, Cervantes O, Jotz GP, Abrahão M. Doenças granulomatosas da Laringe. Acta Awho. 1998;17(1):6-10.
10. World Health Organization (WHO). The Leishmaniases: report of a WHO Expert Committee. Geneva, 1984. (Technical Report Series; 701). 